\title{
Introducing an Innovative Method to Treat Spastic Cerebral Palsy with Faster Recovery Time: Post-Achilles Tendon Lengthening with Bandaging
}

\author{
Hamed Yazdanshenas ${ }^{1,2,3,4,5^{*}}$, Firoozeh Madadi6, Kevin Perez ${ }^{7,8}$, \\ Eleby Rudolph Washington III1,2,3, Arya Nick Shamie ${ }^{2}$, Firooz Madadi ${ }^{9}$
}

\author{
${ }^{1}$ Department of Orthopedic Surgery, College of Medicine, Charles R. Drew University of Medicine and Science (CDU), \\ Los Angeles, USA \\ ${ }^{2}$ Department of Orthopedic Surgery, David Geffen School of Medicine, University of California, Los Angeles (UCLA), \\ Los Angeles, USA \\ ${ }^{3}$ Department of General Surgery, College of Medicine, Charles R. Drew University of Medicine and Science, Los Angeles (CDU), \\ Los Angeles, USA \\ ${ }^{4}$ Department of Family Medicine, David Geffen School of Medicine, University of California, Los Angeles (UCLA), \\ Los Angeles, USA \\ ${ }^{5}$ Department of Family Medicine, College of Medicine, Charles R. Drew University of Medicine and Science, Los Angeles (CDU), \\ Los Angeles, USA \\ ${ }^{6}$ Department of Anesthesiology, Anesthesiology Research Center, Shabid Beheshti University of Medical Sciences (SBMU), \\ Tehran, Iran \\ ${ }^{7}$ College of Medicine, Charles R. Drew University of Medicine and Science (CDU), Los Angeles, USA \\ ${ }^{8}$ David Geffen School of Medicine, University of California, Los Angeles (UCLA), Los Angeles, USA \\ ${ }^{9}$ Department of Orthopedic Surgery, Akhtar Hospital, Shabid Beheshti University of Medical Sciences (SBMU), Tehran, Iran \\ Email: *Yazdanshenas@ucla.edu
}

How to cite this paper: Yazdanshenas, $\mathrm{H}$. Madadi, F., Perez, K., Washington III, E.R., Shamie, A.N. and Madadi, F. (2021) Introducing an Innovative Method to Treat Spastic Cerebral Palsy with Faster Recovery Time: Post-Achilles Tendon Lengthening with Bandaging. Journal of Biosciences and Medicines, 9, 30-39. https://doi.org/10.4236/jbm.2021.912004

Received: October 20, 2021

Accepted: November 30, 2021

Published: December 3, 2021

\begin{abstract}
Equinus deformity is one of the most common musculoskeletal deformities of Cerebral Palsy (CP) which is defined as an abnormality in ankle dorsiflexion that can cause severe walking disability. The standard surgical intervention for Equinus deformity is Achilles Tendon Lengthening (ATL) with postoperative leg casting, however in some cases, it requires months of recovery post-operation to regain movement. The objective of this study was to find an alternative to leg casting that would provide a safe and faster time course to recovery. Therefore, we conducted a semi-experimental clinical trial study of a population that included $62 \mathrm{CP}$ patients between the ages of $6-15$ years of age with diplegia, hemiplegia, or quadriplegia that randomly underwent ATL followed by subsequent leg casting (control, 32 patients) or bandage and ankle-foot orthosis (AFO; experimental, 30 patients). Patients were periodically
\end{abstract}


Copyright $\odot 2021$ by author(s) and Scientific Research Publishing Inc. This work is licensed under the Creative Commons Attribution International License (CC BY 4.0).

http://creativecommons.org/licenses/by/4.0/ evaluated for mobility and walking ability pre-operation and at 2 weeks postoperation, 6 weeks post-operation, and then every month for 6 months. We found that all 32 patients in the experimental group regained their walking ability within two weeks (100\%), while majority of patients in the control group (84\%) regained their walking ability after 4 - 6 weeks, regardless of CP type. We also found that on 6-month follow-up, progression of walking abilities was similar in both groups. Rupture of the repaired tendon was not observed in any of the participants in either group. This study suggests applying a bandage with a foot or ankle orthosis as an alternative to long leg cast in children of 15 years of age or younger with CP treated with ATL for Equinus deformity to facilitate faster post-operative recovery. It also recommends follow-up studies on confounding factors, categorization of pre- and post-operative range of movement, and post-op complications with longer follow up while treating CP patient with ATL followed by AFO.

\section{Keywords}

Cerebral Palsy, Equinus Deformity, Recovery

\section{Introduction}

Cerebral Palsy (CP) is not an individual disorder with a single underlying etiology, but rather an umbrella term with diverse causes and symptomatology, the latter of which can change significantly with age [1] [2]. The definition of CP has gradually adapted to accommodate a wide range of disease conditions. One commonly accepted modern definition describes $\mathrm{CP}$ as a group of permanent but changing disorders that affect movement and posture, or motor function caused by a lesion or deficit of the developing brain [2] [3] [4]. CP with motor function disorders is typically associated with other neurological disorders including sensation-perceptual, cognitive, or behavioral disorders as well as epilepsy and secondary musculoskeletal disorders [1] [5]. The most recent report by the Surveillance of Cerebral Palsy in Europe (SCPE) states that the average rate of CP is 1.5 - 3.0 per 1000 live births in Europe. In the United States, it was reported to be 1.76 per 1000 live births in 2002 [6]. A correlation between birth weight and $\mathrm{CP}$ has also been reported, stating that premature babies weighing under 1500 grams had a 70x higher rate of CP than children weighing 2500 grams or more at birth [1].

Complications can also arise in CP such as epilepsy, intellectual impairment, gastrointestinal problems (GI), and musculoskeletal (MSK) deformities. MSK deformities are quite common in patients with $\mathrm{CP}$ and can cause gait abnormalities, leading to significantly diminished quality of life. These deformities can range from simple toe walking to Equinus deformity, which is the contracture of the gastrocnemius causing persistent plantarflexion of the foot thus preventing the heel from touching the ground while walking [7] [8] [9] [10]. Management of lower limb deformities depends on severity and is usually a step-wise ap- 
proach over the time course of development of the child. Clinical management usually begins with muscle stretching and orthotics to aid in walking. If initial management is ineffective, injections of botulinum toxin can then be used to relax the contracture [11]. Typically, these conservative measures are able to resolve the issue by 6 - 8 years of age with surgical intervention being considered if the Equinus has not resolved [7] [10]. Other complications that should be taken into consideration when managing $\mathrm{CP}$ is the reduction of spasticity. This can be done by introduction of pharmaceuticals into the management plan, such as benzodiazepines or GABA agonists [12]. The standard of care for surgical intervention of Equinus is the Achilles tendon lengthening (ATL) procedure, which is performed by either operating on the tendon or tendon-muscle junction. Generally, the lengthened tendon is post-operatively casted using a long leg cast to protect the surgical site and minimize tendon rupture [7].

Our observations in recent years indicate that some patients have lost their ability to move or walk after the ATL procedure, with some requiring months of recovery post-operation to regain movement in the limb in question. Considering the aim of ATL is to improve the walking abilities of a patient with Equinus, these observations motivated us to find casting-alternatives that could expedite the recovery process and prevent any loss of walking abilities such as non-casting methods. In this study, we compared patients who underwent ATL lengthening and were either placed in a long leg cast or regular bands and band-aids to determine if the alternative of bandaging would expedite recovery and have reduced post-operative injury. The results of this study demonstrate that bandaging as an alternative in the post-operative period can preserve and improve the moving abilities of a patient.

\section{Materials and Methods}

\subsection{Trial Design}

The present study was a semi-experimental clinical trial study in which patients were recruited randomly assigned to either the control or experimental group. Among $168 \mathrm{CP}$ patients who established care at Akhtar Educational Hospital, Pediatric Orthopedic Clinic affiliated by Shahid Beheshti University of Medicine and Science, over a time course of 4 years, 73 patients that met the study selection criteria were invited to join the study with 62 of them agreeing to participate. These 62 patients were recruited after a written informed consent was obtained from the parents and randomly underwent ATL followed by subsequent leg casting (control) or bandage and ankle-foot orthosis (AFO; experimental). The study protocol was approved by Hospital Institutional Review Board, prior to study commencement.

\subsection{Inclusion/Exclusion Criteria}

Criteria for selection included patients between the ages of 6 - 15 years of age with spastic $\mathrm{CP}$ and no other substantial impairments of the limb or spine, no 
notable vision impairment, and no past history of similar Achilles Tendon Lengthening surgeries in the limb on which the operation was planned. Patients were also required to have what the research team considered as "adequate IQ", defined as having any absence of significant or conventional symptoms of mental retardation such as defective recognition of time and location, inability to interact with environment, lack of trainability, or inadequate speech. In this study, we used SCPE's classification which sub-classifies CP into spastic, dyskinetic, and ataxic types. Further evaluation is recommended by the SCPE to evaluate the motor disorders, but was not performed as it was outside the scope of this study [13].

\subsection{Group Allocation}

Patients were designated pre-procedure into the control group (hard cast) or experimental group (bandage) depending on the day in which they were referred to the clinic, and further stratified into age groups $6-10$ and $11-15$ years of age. Patients referred on Wednesday were designated control group, while patients referred on Tuesdays were designated into experimental with parental consent. All patients that met criteria underwent similar open step-cut lengthening of the Achilles tendon.

\subsection{Pre-Operative Care}

Pre-procedure patients were hospitalized for clinical examination by the surgeons. Subjects whose clinical characteristics did not meet the pre-established criteria were omitted from the study. Anesthesiologists examined the patients and listed them for surgery if they were deemed no-risk. General anesthesia was used for both groups, and all anesthesiologists and associates were blinded to the study.

\subsection{Achilles Tendon Lengthening}

All patients underwent a similar ATL (open step-cut lengthening of Achilles tendon) surgical procedure. Patients were placed in supine position while under general anesthesia. Prep and drape were placed and, while under tourniquet pressure, a middle posterior incision was made on the back of the ankle. After opening of the skin, hypodermis, and tendon sheath, the end of the tendon was revealed and divided into the inner and outer halves via a middle incision. Next, the inner and outer halves were separated from the lower and upper portions, respectively. The ankle was then placed at $10^{\circ}-20^{\circ}$ of dorsiflexion and the two halved of the tendons were sutured using 0 nylon stitch thread. Afterwards, the sheath on the tendon was sutured using 3-0 vicryl thread, and the dermis and hypodermis were closed with a sterile bandage placed on the site of incision.

After the surgical procedure, the control group was placed in a long leg cast while the ankle was at $90^{\circ}$ dorsiflexion with the knee at complete extension. The experimental group had a crepe bandage applied at the base of the toes up to be- 
low the knee. The ankle was then placed in a $90^{\circ}$ dorsiflexion using leucoplast sticks and the knee placed in full extension.

\subsection{Post-Operative Care}

Patients were then woken up from anesthesia and sent to the ward under the discretion of the anesthesiologist. Post-operative observation was identical in both groups including diet, hospitalization period, analgesic use, etc. Patients were encouraged to walk once pain was adequately managed and supervised by a chiropractor or physical therapist. Their walking capacity was recorded throughout their stay, and discharged from hospital within 2 - 3 days. Patients and caregivers were educated on continuous walking and bandage application.

\subsection{Outcomes Assessment}

Patients were monitored sequentially after being discharged from the hospital in the outpatient setting. This occurred at 2 weeks post-op, 6 weeks post-op, then every month for 6 months. Both groups were assessed for quality and amount of walking compared to pre-surgery at each interval. At 2 weeks post-op, the experimental group had their bandage opened, stitches removed, and orthosis placed while the control group had their cast opened to reveal the stitches for removal and cast repaired. At 6 weeks post-op, the control group had their casts removed and referred to the chiropractor for improvement of moving and walking status, while the experimental group maintain their orthosis for walking. At the monthly visits, patients were examined for re-rupture of the Achilles tendon.

\subsection{Statistical Analysis}

The data were analyzed and frequency distribution tables were created. The categorical variables included age, gender, CP type, daily activity (pre-op), disorder type, associated deformities, walking time (post-op), and re-rupture of Achilles tendon. These variables were categorized in two groups (Experimental and Control) and three subgroups (Diplegia, Hemiplegia, and Quadriplegia). The data of each group and each subgroup were compared in two combined tables (Table 1, Table 2). The statistical analysis including a Student's t-test was performed with the SPSS ${ }^{\circledR}$ program (SPSS 20.0 for Windows) to determine the correlates of the independent variables. P. Value $<0.05$ was considered statistically significant.

\section{Results}

There was a total of 62 patients that recruited to our study, which were stratified into 32 experimental and 30 control subjects, which were further sub-stratified into patients with diplegia, hemiplegia, or quadriplegia. The experimental group contained 8 (57\%) male and 6 (43\%) female diplegic, 6 (50\%) male and $6(50 \%)$ female hemiplegic, and 4 (67\%) and 2 (33\%) quadriplegic patients. The age groups for experimental contained a greater number of patients in the $6-10$ years of age group, showing $9(64 \%) 6$ - 10 and $5(36 \%) 11$ - 15 years of age diplegic patients, 
7 (58\%) 6 - 10 and 5 (42\%) 11 - 15 years of age hemiplegic patients, and 4 (67\%) 6 - 10 and $2(33 \%) 11$ - 15 years of age quadriplegic patients. The control group showed similar numbers, with 6 - 10 years of age group containing 20 (67\%) and 10 - 15 years of age group containing 10 (33\%); the distribution can be seen on Table 1.

As for frequency of procedure sidedness, in our experimental group 14 (100\%) diplegic patients received a two-sided procedure, $12(100 \%)$ hemiplegic patients received a one-sided procedure, and 6 (100\%) quadriplegic patients received one-sided procedure. Similarly, the control group showed 20 (100\%) of diplegic patients received a two-sided procedure, $6(100 \%)$ hemiplegic patients received a one-sided procedure, and 4 (100\%) quadriplegic patients received a two-sided procedure. Walking status prior to corrective procedure included a larger number of patients walking without aids in both experimental $(20 ; 63 \%)$ and control (18; 60\%) groups. The experimental group showed that 8 (57\%) diplegic, 9 (75\%) hemiplegic, and 3 (50\%) quadriplegic patients were walking without aids prior to their corrective procedure. Similarly, in the control group 12 (60\%) of diplegic, 4 (67\%) hemiplegic, and 2 (50\%) quadriplegic patients were walking without aids; the remainder of the frequency of distribution can be seen on $\mathrm{Ta}$ ble 1 .

Table 1. Frequency distribution of the studied patients' age, gender, CP type, and daily activity in both experimental and control groups and their subgroups.

\begin{tabular}{|c|c|c|c|c|c|c|c|c|}
\hline & \multicolumn{4}{|c|}{ Experimental Group } & \multicolumn{4}{|c|}{ Control Group } \\
\hline & \multirow{2}{*}{$\frac{\text { Diplegia }}{f(\%)}$} & \multicolumn{2}{|c|}{ Hemiplegia Quadriplegia } & \multirow{2}{*}{$\begin{array}{l}\text { Total } \\
f(\%)\end{array}$} & \multirow{2}{*}{$\begin{array}{c}\text { Diplegia } \\
f(\%)\end{array}$} & \multicolumn{2}{|c|}{ Hemiplegia Quadriplegia } & \multirow{2}{*}{$\begin{array}{l}\text { Total } \\
f(\%)\end{array}$} \\
\hline & & $f(\%)$ & $f(\%)$ & & & $f(\%)$ & $f(\%)$ & \\
\hline \multicolumn{9}{|c|}{ Gender } \\
\hline Male & $8(57)$ & $6(50)$ & $4(67)$ & $18(57)$ & $13(65)$ & $4(67)$ & $2(50)$ & $19(63)$ \\
\hline Female & $6(43)$ & $6(50)$ & $2(33)$ & $14(43)$ & $7(35)$ & $2(33)$ & $2(50)$ & $11(37)$ \\
\hline \multicolumn{9}{|c|}{ Age } \\
\hline $6-10$ & $9(64)$ & $7(58)$ & $4(67)$ & $20(62)$ & $14(70)$ & $3(50)$ & $3(75)$ & $20(67)$ \\
\hline $11-15$ & $5(36)$ & $5(42)$ & $2(33)$ & $12(38)$ & $6(30)$ & $3(50)$ & $1(25)$ & $10(33)$ \\
\hline \multicolumn{9}{|c|}{ Type } \\
\hline One-Sided & $0(0)$ & $12(100)$ & $0(0)$ & $12(38)$ & $0(0)$ & $6(100)$ & $0(0)$ & $6(20)$ \\
\hline Two-Sided & $14(100)$ & $0(100)$ & $6(100)$ & $20(62)$ & $20(100)$ & $0(0)$ & $4(100)$ & $24(80)$ \\
\hline \multicolumn{9}{|c|}{ Walking status } \\
\hline Not standing & $0(0)$ & $0(0)$ & $0(0)$ & $0(0)$ & $0(0)$ & $0(0)$ & $1(25)$ & $1(3)$ \\
\hline Standing (and walking-limited) & $0(0)$ & $0(0)$ & $1(16.7)$ & $1(3)$ & $0(0)$ & $0(0)$ & $0(0)$ & $0(0)$ \\
\hline Walking with parents' help & $2(14)$ & $1(8)$ & $1(16.7)$ & $4(12)$ & $0(0)$ & $0(0)$ & $0(0)$ & $0(0)$ \\
\hline Walking with orthopedic aids & $4(29)$ & $2(17)$ & $1(16.7)$ & $7(22)$ & $8(40)$ & $2(33)$ & $1(25)$ & $11(37)$ \\
\hline Walking without aids & $8(57)$ & $9(75)$ & $3(50)$ & $20(63)$ & $12(60)$ & $4(67)$ & $2(50)$ & $18(60)$ \\
\hline Total & $14(44)$ & $12(37)$ & $6(19)$ & $32(100)$ & $20(67)$ & $6(20)$ & $4(13)$ & $30(100)$ \\
\hline
\end{tabular}


The distribution of onset of walking showed significant difference $(\mathrm{p}=0.048)$ between the experimental and control groups at 2 weeks follow-up post-operation, however the difference was not significant at 6 weeks $(p>0.05)$. Also, our data did not show significant difference between CP sub-types including diplegia, hemiplegia, and quadriplegia ( $p>0.05$ ). Everyone in our experimental group was found to be walking within 2 weeks, with the majority being 10 (72\%) diplegic and 10 (83\%) hemiplegic patients walking within 1-week post-procedure and $4(67 \%)$ quadriplegic patients walking within 2 weeks post-procedure. On the other hand, 14 (70\%) diplegic and $2(50 \%)$ quadriplegic patients in our control group began walking within 6 weeks post procedure and 4 (66\%) hemiplegic patients began walking between 3 - 5 weeks. The remainder of patient frequency distribution can be seen on Table 2. It should also be noted that both experimental and control groups did not have rupture of the Achilles tendon on all follow-ups up to 6 months. The distribution of associated deformities in the experimental group included 4 knee, 5 hip, 1 spine, and 6 hand deformities. For the control group, these included 4 knee, 7 hip, 2 spine, and 1 hand deformities.

\section{Discussion}

Equinus deformity is defined as the inability to create contact between the heel and underlying surface while in ankle dorsiflexion without proper compensation of the lower limb that can be a debilitating contracture of the lower limb if not properly managed [14]. The importance of treating Equinus deformity is to provide increased quality of life which includes ability to walk without the need for aids, resolving deformity, and relieving pain. The standard of care currently is to perform an Achilles Tendon Lengthening (ATL) surgery with post-operative leg casting, however in some cases it requires months of recovery post-operation to regain movement.

The objective of this study was to find a non-casting alternative to leg casting

Table 2. Frequency distribution of walking time post-operation of patients from the experimental and control groups and their subgroups.

\begin{tabular}{|c|c|c|c|c|c|c|c|c|}
\hline & \multicolumn{4}{|c|}{ Experimental Group } & \multicolumn{4}{|c|}{ Control Group } \\
\hline & Diplegia & Hemiplegia & Quadriplegia & Total & Diplegia & Hemiplegia & Quadriplegia & Total \\
\hline & $f(\%)$ & $f(\%)$ & $f(\%)$ & $f(\%)$ & $f(\%)$ & $f(\%)$ & $f(\%)$ & $f(\%)$ \\
\hline Less than 1 week & $10(72)$ & $10(83)$ & $2(33)$ & $22(69)$ & $0(0)$ & $0(0)$ & $0(0)$ & $0(0)$ \\
\hline 1 - 2 weeks & $4(28)$ & $2(17)$ & $4(67)$ & $10(31)$ & $0(0)$ & $1(17)$ & $0(0)$ & $1(3)$ \\
\hline 2 - 3 weeks & $0(0)$ & $0(0)$ & $0(0)$ & $0(0)$ & $0(0)$ & $0(0)$ & $0(0)$ & $0(0)$ \\
\hline 3 - 4 weeks & $0(0)$ & $0(0)$ & $0(0)$ & $0(0)$ & $1(5)$ & $2(33)$ & $1(25)$ & $4(13)$ \\
\hline 4 - 5 weeks & $0(0)$ & $0(0)$ & $0(0)$ & $0(0)$ & $5(25)$ & $2(33)$ & $1(25)$ & $8(17)$ \\
\hline 5 - 6 weeks & $0(0)$ & $0(0)$ & $0(0)$ & $0(0)$ & $14(70)$ & $1(17)$ & $2(50)$ & $17(57)$ \\
\hline
\end{tabular}


that would provide a safe and faster time course to recovery. Therefore, we conducted a semi-experimental clinical trial study of a population that included 62 Cerebral Palsy patients between the ages of $6-15$ years of age with diplegia, hemiplegia, or quadriplegia that randomly underwent ATL followed by subsequent leg casting (control) or bandage and ankle-foot orthosis (AFO; experimental). Interestingly the findings of this study demonstrated that patients who suffered from Cerebral Palsy were likely to regain walking ability earlier if managed post-Achilles tendon lengthening procedure with bandaging and AFO rather than a long leg cast. The 32 patients who did undergo bandaging and AFO regained walking ability within the first two weeks with no serious obstacles for movement. On the other hand, the 30 patients who underwent long leg casting were more likely to regain walking ability much later, at 4 - 6 weeks.

Other studies have discussed the length of the immobilization period as well as types of casting, with differing recommendations on how long the immobilization period should be. The standard of care after ATL surgery is to place the operated-on limb in a hard leg cast with the knee fully extended, and ankle/foot placed in a $90^{\circ}$ dorsiflexed position with major consensus stating to have an immobilization period somewhere between 3 - 6 weeks [15] [16] [17]. This recommendation is about the same length of time that our control group took for them to regain walking ability.

There has also been studies that state regaining walking ability at an earlier time course is beneficial for long term outcomes [18]. Though our study only had a follow-up period of 6 months post-op, none of our patients in either group had any complications that included tendon rupture, re-contracture, or loss of walking ability. Some studies have shown that long-term outcome complications begin forming years later, and so a longer follow-up would be beneficial for us to strengthen our case of using bandaging and AFO over hard leg casting. In a comprehensive systematic review of patients with $\mathrm{CP}$ who underwent ATL, it was reported that the variance of outcomes was based on factors that included method of ATL used, age of patients and time of follow-up [19]. The study also reported that $\mathrm{CP}$ type played into recurrence of Equinus deformity, with a stronger correlation being in spastic type compared to surgical method used. This was also seen in a study conducted by Borton, which showed that a calcaneus gait leading to a crouch gait was seen with higher rates in patients with spastic diplegia/quadriplegia CP type [20]. Similarly, a case series reported that the best outcomes post-ATL occur in patients with hemiplegia type or patients who underwent only a single-limb operation [21].

Considering that the scope of our study was to distinguish the benefit of using bandaging and AFO rather than hard leg casting, our study does have some limitations to it. Our study focused on walking status post-procedure, and so when categorizing walking ability, we did not focus on the classifications of range of motion, pre-operative daily activity, walking time post-procedure, re-rupture of Achilles tendon or other confounding factors that may affect the regaining of 
walking ability. Distinguishing these confounding factors may have been able to explain the discrepancy in length to regaining walking ability between our control and experimental, though all of our experimental cohorts regained walking within two weeks. Another limitation was length of follow-up, which we mentioned to be short in length at six months post-procedure considering some studies show a minimum of two years post-procedure [15] [16] [17] [22]. As previously stated, some studies have shown that complications post-ATL usually occur years later. These considerations would be able to be addressed for future directions of this project.

\section{Conclusion}

This study suggests applying a bandage with a foot or ankle orthosis as an alternative to long leg cast in children of 15 years of age or younger with $\mathrm{CP}$ treated with ATL for Equinus deformity to facilitate faster post-operative recovery. It also recommends follow-up studies on confounding factors, categorization of pre- and post-operative range of movement, and post-op complications with longer follow up while treating CP patient with ATL followed by AFO.

\section{Conflicts of Interest}

The authors declare no conflicts of interest regarding the publication of this paper.

\section{References}

[1] Sadowska, M., Sarecka-Hujar, B. and Kopyta, I. (2020) Cerebral Palsy: Current Opinions on Definition, Epidemiology, Risk Factors, Classification and Treatment Options. Neuropsychiatric Disease and Treatment, 16, 1505-1518. https://doi.org/10.2147/NDT.S235165

[2] Patel, D.R., Neelakantan, M., Pandher, K. and Merrick J. (2020) Cerebral Palsy in Children: A Clinical Overview. Translational Pediatrics, 9, S125-S135.

[3] Bax, M., Goldstein, M., Rosenbaum, P., Leviton, A., Paneth, N., Dan, B., et al. (2005) Proposed Definition and Classification of Cerebral Palsy, April 2005. Developmental Medicine \& Child Neurology, 47, 571-576. https://doi.org/10.1017/S001216220500112X

[4] Christine, C., Dolk, H., Platt, M.J., Colver, A., Prasauskiene, A., Krageloh-Mann, I., et al. (2007) Recommendations from the SCPE Collaborative Group for Defining and Classifying Cerebral Palsy. Developmental Medicine \& Child Neurology, 109, 35-38. https://doi.org/10.1111/j.1469-8749.2007.tb12626.x

[5] Ikeudenta, B.A. and Rutkofsky, I.H. (2020) Unmasking the Enigma of Cerebral Palsy: A Traditional Review. Cureus, 12, e11004. https://doi.org/10.7759/cureus.11004

[6] Van Naarden Braun, K., Doernberg, N., Schieve, L., Christensen, D., Goodman, A. and Yeargin-Allsopp, M. (2016) Birth Prevalence of Cerebral Palsy: A PopulationBased Study. Pediatrics, 137, e20152872. https://doi.org/10.1542/peds.2015-2872

[7] Sees, J.P. and Miller, F. (2013) Overview of Foot Deformity Management in Children with Cerebral Palsy. Journal of Children's Orthopaedics, 7, 373-377. https://doi.org/10.1007/s11832-013-0509-4

[8] Hasler, C. and Brunner, R. (2020) Spine Deformities in Patients with Cerebral Palsy. 
Journal of Children's Orthopaedics, 14, 9-16. https://doi.org/10.1302/1863-2548.14.190139

[9] Lieber, R.L. and Friden, J. (2019) Muscle Contracture and Passive Mechanics in Cerebral Palsy. Journal of Applied Physiology, 126, 1492-1501.

https://doi.org/10.1152/japplphysiol.00278.2018

[10] Mathewson, M.A. and Lieber, R.L. (2015) Pathophysiology of Muscle Contractures in Cerebral Palsy. Physical Medicine and Rehabilitation Clinics of North America, 26, 57-67. https://doi.org/10.1016/j.pmr.2014.09.005

[11] Fehlings, D., Brown, L., Harvey, A., Himmelmann, K., Lin, J.P., Macintosh, A., et al. (2018) Pharmacological and Neurosurgical Interventions for Managing Dystonia in Cerebral Palsy: A Systematic Review. Developmental Medicine \& Child Neurology, 60, 356-366. https://doi.org/10.1111/dmcn.13652

[12] Haque, N., Saha, N., Ahmed, T., Debnath, B., Mollah, A. and Rahman, E. (2020) Comparison of Baclofen and Tizanidine in Reducing Spasticity in Cerebral Palsy: A Randomized Control Trial. Open Journal of Pediatrics, 10, 617-625. https://doi.org/10.4236/ojped.2020.104063

[13] Paulson, A. and Vargus-Adams, J. (2017) Overview of Four Functional Classification Systems Commonly Used in Cerebral Palsy. Children, 4, Article No. 30. https://doi.org/10.3390/children4040030

[14] Cobeljic, G., Bumbasirevic, M., Lesic, A. and Bajin, Z. (2009) The Management of Spastic Equinus in Cerebral Palsy. Orthopaedics and Trauma, 23, 201-209. https://doi.org/10.1016/j.mporth.2009.05.003

[15] Lee, C.L. and Bleck, E.E. (1980) Surgical Correction of Equinus Deformity in Cerebral Palsy. Developmental Medicine \& Child Neurology, 22, 287-292. https://doi.org/10.1111/j.1469-8749.1980.tb03707.x

[16] Moreau, M.J. and Lake D.M. (1987) Outpatient Percutaneous Heel Cord Lengthening in Children. Journal of Pediatric Orthopaedics, 7, 253-255. https://doi.org/10.1097/01241398-198705000-00001

[17] Rattey, T.E., Leahey, L., Hyndman, J., Brown, D.C. and Gross, M. (1993) Recurrence after Achilles Tendon Lengthening in Cerebral Palsy. Journal of Pediatric Orthopaedics, 13, 184-187.

[18] Katz, K., Arbel, N., Apter, N. and Soudry, M. (2000) Early Mobilization after Sliding Achilles Tendon Lengthening in Children with Spastic Cerebral Palsy. Foot \& Ankle International, 21, 1011-1014. https://doi.org/10.1177/107110070002101205

[19] Shore, B.J., White, N. and Kerr Graham, H. (2010) Surgical Correction of Equinus Deformity in Children with Cerebral Palsy: A Systematic Review. Journal of Children's Orthopaedics, 4, 277-290. https://doi.org/10.1007/s11832-010-0268-4

[20] Borton, D.C., Walker, K., Pirpiris, M., Nattrass, G.R. and Graham, H.K. (2001) Isolated Calf Lengthening in Cerebral Palsy. Outcome Analysis of Risk Factors. The Journal of Bone and Joint Surgery, 83, 364-370. https://doi.org/10.1302/0301-620X.83B3.0830364

[21] Dietz, F.R., Albright, J.C. and Dolan, L. (2006) Medium-Term Follow-Up of Achilles Tendon Lengthening in the Treatment of Ankle Equinus in Cerebral Palsy. Iowa Orthopedic Journal, 26, 27-32.

[22] Joo, S.Y., Knowtharapu, D.N., Rogers, K.J., Holmes, L. and Miller, F. (2011) Recurrence after Surgery for Equinus Foot Deformity in Children with Cerebral Palsy: Assessment of Predisposing Factors for Recurrence in a Long-Term Follow-Up Study. Journal of Children's Orthopaedics, 5, 289-296.

https://doi.org/10.1007/s11832-011-0352-4 